\title{
Cerebral Venous Thrombosis Associated with COVID-19 Infection: An Observational, Multicenter Study
}

\author{
Sajid Hameed ${ }^{a}$ Mohammad Wasay ${ }^{a}$ Bashir A. Soomro ${ }^{b}$ Ossama Mansour ${ }^{c}$ \\ Foad Abd-allah ${ }^{d}$ Tianming Tu $^{\mathrm{e}}$ Raja Farhat $^{f}$ Naila Shahbaz $^{\mathrm{g}}$ \\ Husnain Hashim $^{\mathrm{h}}$ Wasim Alamgir ${ }^{\mathrm{i}}$ Athar lqbal $^{\mathrm{j}}$ Maria Khan $^{\mathrm{k}}$
}

${ }^{a}$ Department of Medicine, Aga Khan University, Karachi, Pakistan; ${ }^{b}$ Department of Neurology, Ziauddin University

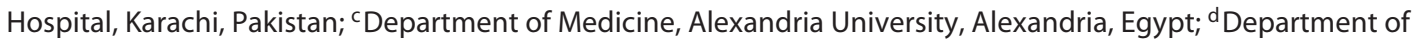

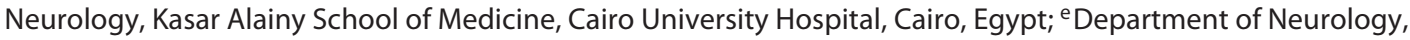
National Neuroscience Institute, Singapore, Singapore; ${ }^{\mathrm{f} S h i f a}$ International Hospital, Islamabad, Pakistan; ${ }^{9}$ Dow University of Health Sciences, Karachi, Pakistan; hFouji Foundation Hospital, Rawalpindi, Pakistan; 'Military Hospital, Rawalpindi, Pakistan; jSheikh Zayed Federal Postgraduate Medical Institute/Hospital, Lahore, Pakistan; ${ }^{\mathrm{k}}$ Rashid Hospital, Dubai, UAE

\section{Keywords}

COVID-19 · Cerebral venous thrombosis · Hemorrhage ·

Coagulopathy $\cdot$ Stroke

\begin{abstract}
Background and Purpose: Coronavirus disease 2019 (COVID-19) has an increased propensity for systemic hypercoagulability and thromboembolism. An association with cerebrovascular diseases, especially cerebral venous thrombosis $(\mathrm{CVT})$, has been reported among these patients. The objective of the present study was to identify risk factors for CVT as well as its presentation and outcome in COVID-19 patients. Methods: This is a multicenter and multinational observational study. Ten centers in 4 countries (Pakistan, Egypt, Singapore, and the United Arab Emirates) participated in this study. The study included patients (aged $>18$ years) with symptomatic CVT and recent COVID-19 infection. Results: Twenty patients (70\% men) were included. Their mean age was 42.4 years, with a male-to-female ratio of 2.3:1. Headache $(85 \%)$ and seizures (65\%) were the common presenting
\end{abstract}

karger@karger.com www.karger.com/cee

Karger"

BOPEN ACCESS
(C) 2021 The Author(s)

Published by S. Karger AG, Basel

This is an Open Access article licensed under the Creative Commons Attribution-NonCommercial-4.0 International License (CC BY-NC) (http://www.karger.com/Services/OpenAccessLicense), applicable to the online version of the article only. Usage and distribution for commercial purposes requires written permission. symptoms, with a mean admission Glasgow Coma Scale (GCS) score of 13. CVT was the presenting feature in 13 cases (65\%), while 7 patients (35\%) developed CVT while being treated for COVID-19 infection. Respiratory symptoms were absent in $45 \%$ of the patients. The most common imaging finding was infarction (65\%), followed by hemorrhage (20\%). The superior sagittal sinus (65\%) was the most common site of thrombosis. Acute inflammatory markers were raised, including elevated serum D-dimer (87.5\%), erythrocyte sedimentation rate $(69 \%)$, and C-reactive protein $(47 \%)$ levels. Homocysteine was elevated in half of the tested cases. The mortality rate was $20 \%$ (4 patients). A good functional outcome was seen in the surviving patients, with a mean modified Rankin Scale score at discharge of 1.3. Nine patients (45\%) had a modified Rankin Scale score of $0-1$ at discharge. Conclusion: COVID-19-related CVT is more common among males at older ages when compared to previously reported non-COVID-19-related CVT cases. CVT should be suspected in COVID-19 patients presenting with headache or seizures. Mortality is high, but functional neurological outcome is good among survivors.

(c) 2021 The Author(s)

Published by S. Karger AG, Basel
Correspondence to:

Mohammad Wasay, mohammad.wasay@aku.edu 


\section{Introduction}

Coronavirus disease 2019 (COVID-19) is a novel infection caused by the SARS-CoV-2 coronavirus, resulting in severe acute respiratory syndrome (SARS). An increased propensity for systemic hypercoagulability and thromboembolism has been reported among COVID-19 patients [1-3]. In one study, thrombotic complications were reported in approximately one-third of the critically ill COVID-19 patients [1]. An increase in cerebrovascular diseases (1-6\%) has also been reported among these patients [4]. Published data on cerebral venous thrombosis (CVT) among COVID-19 patients are limited. Hughes et al. [5] published the first case of CVT in a COVID-19 patient in April 2020. To the best of our knowledge, only 12 CVT cases among COVID-19 patients have been reported in the literature [6]. All these cases are presented in single-center case reports. The purpose of this study was to identify the risk factors for CVT as well as its presentation and outcome in COVID-19 patients in a multinational, multicenter setting using a prespecified data collection tool.

\section{Subjects and Methods}

We conducted a multicenter observational study. Ten centers in 4 countries (Pakistan, Egypt, Singapore, and the United Arab Emirates) participated in the study. All 10 centers are tertiary care hospitals with full-time neurologists and availability of diagnostic modalities for the diagnosis of CVT as well as COVID-19. Data were collected on a prespecified questionnaire. They were collected retrospectively from COVID-19 registries and hospital medical records. All patients fulfilling the inclusion criteria were included. One male patient with recent head trauma with COVID-19 and CVT was excluded. Patients aged 18 years or above with recent COVID-19 infection, confirmed either by reverse transcriptase-polymerase chain reaction assay of a nasopharyngeal swab or serum antibody testing for COVID-19, were included. Diagnosis of CVT was confirmed by at least one of the following imaging studies according to the established criteria: magnetic resonance (MR) imaging, MR venography (MRV), computed tomography (CT), CT venography, or cerebral venography. Patients with recent trauma and patients already receiving anticoagulation were excluded. The data included risk factors, clinical features, laboratory findings, imaging findings, COVID-19-related information, and data on management and outcome for all patients enrolled in the study. The patients were identified through the medical record system of the hospitals and COVID-19 registries at the various centers. Data are presented as frequencies and correlations.

\section{Results}

A total of 20 cases were included (Table 1) from 4 countries (13 from Pakistan, 4 from Egypt, 2 from Singapore, and 1 from the United Arab Emirates). Eighteen cases $(90 \%)$ tested positive for SARS-CoV-2 by reverse transcriptase-polymerase chain reaction assay of a nasopharyngeal swab, while 2 cases $(10 \%)$ tested positive for COVID-19 antibodies. Out of 20 subjects, 14 were male (70\%). The average age was 42.4 years. In 19 cases (95\%), the diagnosis of CVT was confirmed by MRV and/or CT venography, while 1 patient had a noncontrast CT scan suggestive of superior sagittal sinus thrombosis.

\section{Clinical Features, Risk Factors, and Comorbid Conditions}

Headache $(85 \%, n=17)$ and seizures $(65 \%, n=13)$ were the most common neurological features. Headache was of 3-7 days in duration in $76 \%$ of the cases. Seizures were of generalized onset in 10 cases (77\%) and focal onset in 3 cases $(23 \%)$. The mean Glasgow Coma Scale (GCS) score on admission was 13. Cigarette-smoking $(45 \%, n=9)$ and hypertension $(35 \%, n=7)$ were the most common vascular risk factors present. One female was on oral contraceptives despite a previous history of CVT. One female had a history of deep venous thrombosis, and 1 male had polycythemia. Four cases were dehydrated (20\%) on presentation.

\section{Relationship to COVID-19 Infection}

CVT was the presenting feature in 13 (65\%) of cases, whereas 7 (35\%) developed CVT while being treated for COVID-19 infection. Two patients with positive COVID-19 antibodies likely presented with late-stage COVID-19. Nine cases (45\%) did not have respiratory symptoms at the time of diagnosis of CVT. In 5 patients $(25 \%)$, CVT was the sole presentation of COVID-19 (absence of fever and respiratory symptoms).

\section{Laboratory Workup and Imaging Findings}

Important laboratory findings included elevated levels of serum D-dimer (87.5\%), erythrocyte sedimentation rate $(69 \%)$, and leukocytosis $(72 \%)$. Homocysteine was elevated in $50 \%$ of the tested cases. Two out of 9 patients had positive lupus anticoagulant (LA). The most common imaging finding was infarction $(65 \%, n=13)$, more than one-third of which were hemorrhagic infarcts $(38 \%$, $n=5)$, followed by hemorrhage only in 4 patients $(20 \%)$. The superior sagittal sinus was the most common site of thrombosis $(65 \%, n=13)$ using all modalities. 


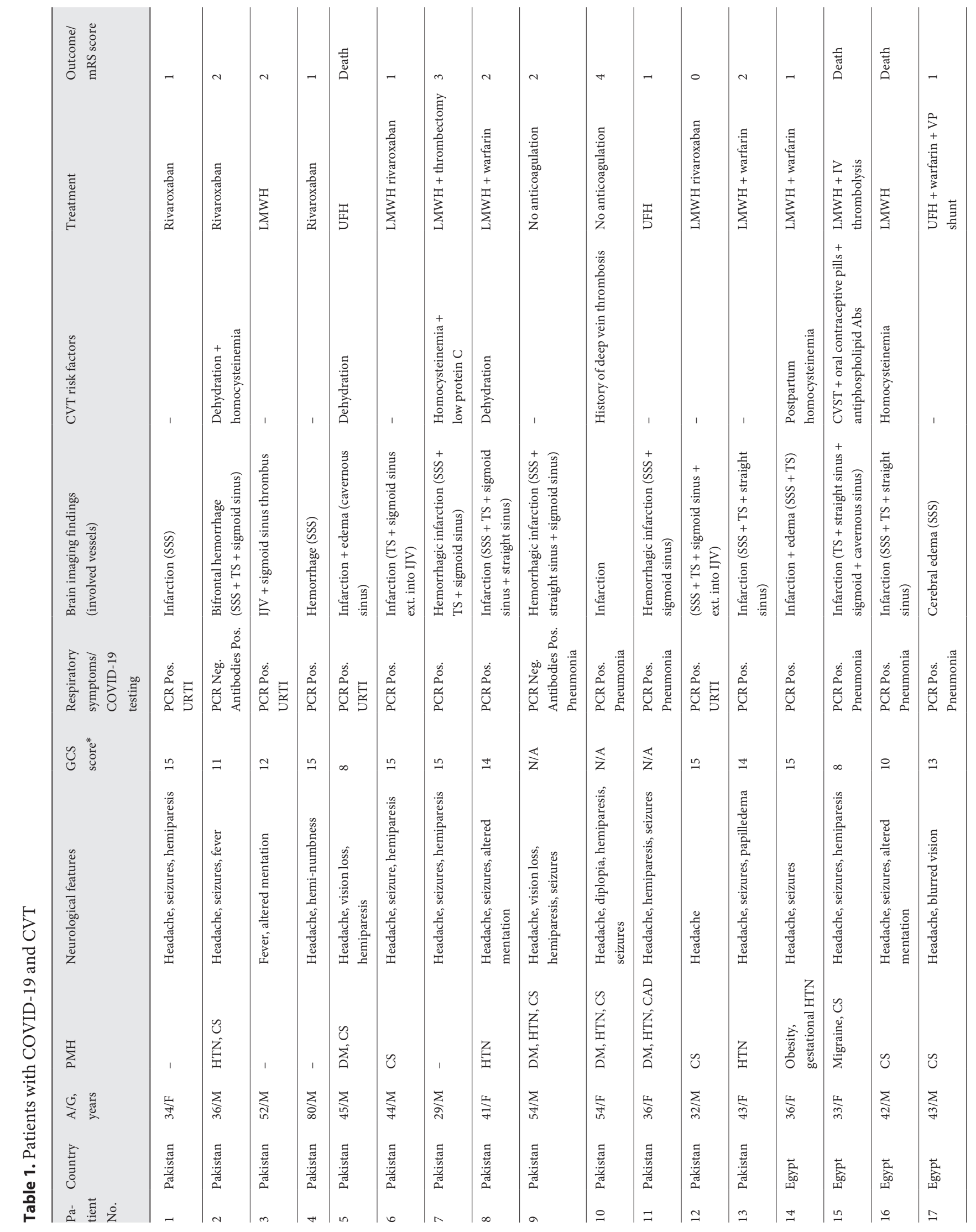




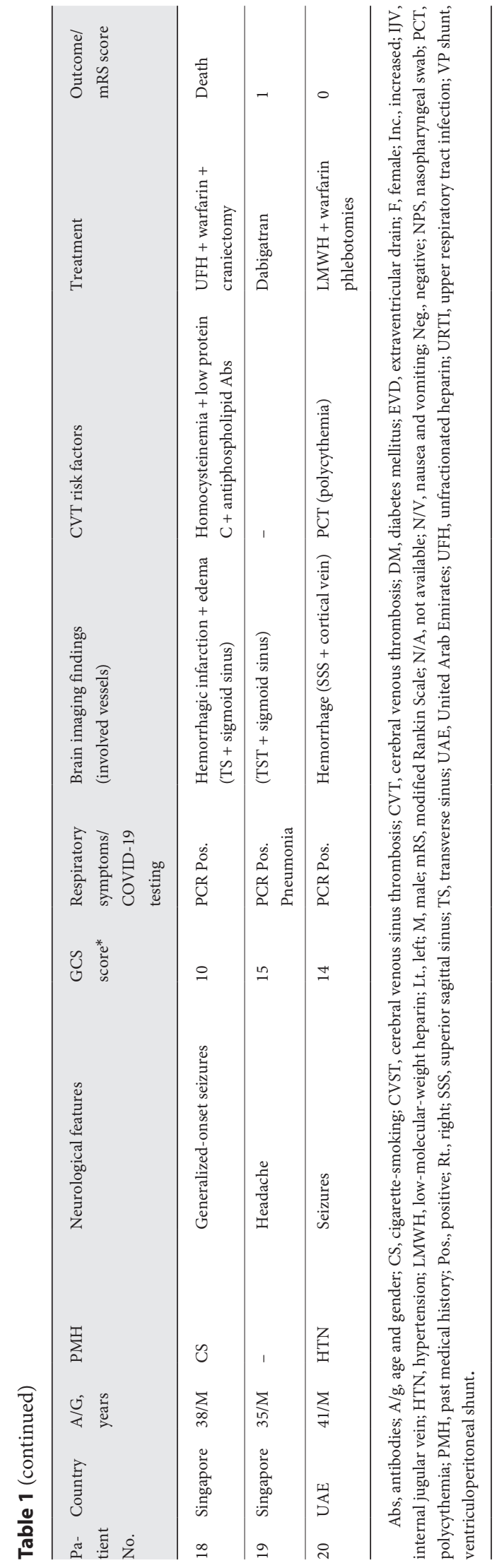

\section{Management and Outcome}

Eighteen cases (90\%) received systemic anticoagulation treatment, with the majority of the patients receiving low-molecular-weight heparin $(56 \%, n=10)$ and unfractionated heparin $(22 \%, n=4)$ for initial anticoagulation. Besides anticoagulation, thrombectomy, intravenous thrombolysis, ventriculoperitoneal shunt, and decompressive craniectomy with extraventricular drain placement were performed on 1 patient each.

Fifteen cases (75\%) were discharged home with an improving condition, 4 cases (20\%) died, and 1 case (5\%) was referred to another hospital for rehabilitation. All 4 patients who died had a low GCS score (8-10) at admission. The discharged cases had an average modified Rankin Scale score of 1.3. Patients with good functional outcome had no or mild respiratory symptoms, a high GCS score (13-15) at admission, and/or a normal brain parenchyma on imaging.

\section{Discussion}

This is the first and the largest multicenter and multinational study of CVT in COVID-19 patients. This patient population differs in many aspects from previously reported non-COVID-19 CVT patients, where CVT is more common in the third decade of life with a female predominance [7-9]. In this study, males were twice as frequently affected, with an average age of 42.4 years. Although the incidence of CVT increases during the puerperal period and is associated with oral contraceptive pill (OCP) use (11-54\%) [7-11], in our study only 1 patient was in the puerperal period (3 days after cesarean section) and 1 patient had a history of OCP use.

A hypercoagulable state is an important and wellknown risk factor for CVT [10-14]. In a study among critically ill COVID- 19 patients $(n=184), 31 \%$ of the patients developed thrombotic complications, with pulmonary embolism topping the list [1]. Similarly, another study reported a high frequency of venous thromboembolism (21\%) among COVID-19 patients [2]. An increased LA positivity is reported in acute COVID-19 infections $[3,12,13]$. These LA antibodies are presumed to increase the risk of thromboembolism but are transiently elevated in the acute viral setting [14]. A prolonged activated partial thromboplastin time in the presence of LA does not increase the bleeding risk, and these patients may receive anticoagulation if clinically indicated [15]. In our study, 9 cases (45\%) also did not have respiratory symptoms. Tan et al. [12] reported an absence of respira- 
tory symptoms in $24 \%$ of their COVID-19 patients with acute ischemic stroke.

SARS-CoV-2, responsible for COVID-19 infection, is well known to bind angiotensin-converting enzyme (ACE) 2 receptors, which are variably present in the arterial and venous vascular endothelium. Autopsy studies have revealed that ACE2 receptors are more abundant in the arterial endothelium than in the venular endothelium [16]. This expression of ACE receptors probably explains the high frequency of arterial stroke. COVID-19 not only causes direct vascular damage but may also create an intense inflammatory reaction affecting hemostasis and the coagulation cascade. COVID-19-associated arterial stroke predominantly affects younger males with vascular risk factors and mostly presents as large vessel stroke [17]. These findings may suggest a common pathway for both COVID-19-associated CVT and arterial stroke.

The main limitations of our study are selection bias, the absence of a control sample, the absence of prothrombotic workup, limited neurological examination, and the inability to examine the influence of confounding variables on outcomes. The current number of CVT cases is probably an underestimation. COVID-19 testing may be included as standard workup among all CVT patients, including those who have no throat or respiratory symptoms. All COVID-19-positive patients with headache and neurological symptoms should be evaluated for CVT. Early diagnosis by MRI and MRV and anticoagulation for high-risk populations (with a history of venous thromboembolism, a postpartum state, or use of OCPs) may lead to improved outcomes.

\section{Statement of Ethics}

The study protocol was approved by the Ethics Review Committees of Aga Khan University, Karachi (Ref. 2020-5204-11458), and the participating centers. The research was conducted ethically in accordance with the World Medical Association Declaration of Helsinki. All participants or their guardians have given written informed consent.

\section{Conflict of Interest Statement}

The authors have no conflict of interest to declare.

\section{Funding Sources}

There are no funding sources to declare.

\section{Author Contributions}

S.H.: data acquisition, data analysis, manuscript writing, and manuscript review. M.W.: concept, collaboration, data acquisition, data analysis, manuscript writing, and manuscript review. B.A.S., O.M., F.A., T.T., R.F., N.S., H.H., W.A., A.I., and M.K.: data acquisition, manuscript writing, and manuscript review.

\section{Data Availability Statement}

All research data are available and can be accessed via the corresponding author.

\section{References}

1 Klok FA, Kruip MJ, van der Meer NJ, Arbous MS, Gommers DA, Kant KM, et al. Incidence of thrombotic complications in critically ill ICU patients with COVID-19. Thromb Res. $2020 \mathrm{Jul} ; 191: 145-7$.

2 Lodigiani C, Iapichino G, Carenzo L, Cecconi M, Ferrazzi P, Sebastian T, et al.; Humanitas COVID-19 Task Force. Venous and arterial thromboembolic complications in COVID 19 patients admitted to an academic hospital in Milan, Italy. Thromb Res. 2020 Jul;191:914.

3 Helms J, Tacquard C, Severac F, Leonard-Lorant I, Ohana M, Delabranche X, et al.; CRICS TRIGGERSEP Group (Clinical Research in Intensive Care and Sepsis Trial Group for Global Evaluation and Research in Sepsis). High risk of thrombosis in patients with severe SARS-CoV-2 infection: a multicenter prospective cohort study. Intensive Care Med. 2020 Jun;46(6):1089-98.
4 Aggarwal G, Lippi G, Michael Henry B. Cerebrovascular disease is associated with an increased disease severity in patients with Coronavirus Disease 2019 (COVID-19): a pooled analysis of published literature. Int J Stroke. 2020 Jun;15(4):385-9.

5 Hughes C, Nichols T, Pike M, Subbe C, Elghenzai S. Cerebral Venous Sinus Thrombosis as a Presentation of COVID-19. Eur J Case Rep Intern Med. 2020 Apr;7(5):001691.

6 Hameed S, Wasay M. Cerebral Venous Sinus Thrombosis Associated with Coronavirus Infection (COVID-19). Pakistan J Neurol Sci. 2020;15(3):60-5.

7 Wasay M, Kaul S, Menon B, Dai AI, Saadatnia M, Malik A, et al. Asian Study of Cerebral Venous Thrombosis. J Stroke Cerebrovasc Dis. 2019 Oct;28(10):104247.
8 Duman T, Uluduz D, Midi I, Bektas H, Kablan Y, Goksel BK, et al.; VENOST Study Group. A multicenter study of 1144 patients with cerebral venous thrombosis: the VENOST study. J Stroke Cerebrovasc Dis. 2017 Aug;26(8):1848-57.

9 Ferro JM, Canhão P, Stam J, Bousser MG, Barinagarrementeria F; ISCVT Investigators. Prognosis of cerebral vein and dural sinus thrombosis: results of the International Study on Cerebral Vein and Dural Sinus Thrombosis (ISCVT). Stroke. 2004 Mar;35(3):664-70.

10 Khealani BA, Wasay M, Saadah M, Sultana E, Mustafa S, Khan FS, et al. Cerebral venous thrombosis: a descriptive multicenter study of patients in Pakistan and Middle East. Stroke. 2008 Oct;39(10):2707-11. 
11 Narayan D, Kaul S, Ravishankar K, Suryaprabha T, Bandaru VC, Mridula KR, et al. Risk factors, clinical profile, and long-term outcome of 428 patients of cerebral sinus venous thrombosis: insights from Nizam's Institute Venous Stroke Registry, Hyderabad (India). Neurol India. 2012 Mar-Apr;60(2): 154-9.

12 Tan YK, Goh C, Leow AS, Tambyah PA, Ang A, Yap ES, et al. COVID-19 and ischemic stroke: a systematic review and meta-summary of the literature. J Thromb Thrombolysis. 2020 Oct;50(3):587-95.
13 Bowles L, Platton S, Yartey N, Dave M, Lee K, Hart DP, et al. Lupus Anticoagulant and Abnormal Coagulation Tests in Patients with Covid-19. N Engl J Med. 2020 Jul;383(3):28890.

14 Devreese KM, Linskens EA, Benoit D, Peperstraete $\mathrm{H}$. Antiphospholipid antibodies in patients with COVID-19: a relevant observation? J Thromb Haemost. 2020 Sep;18(9): 2191-201.

15 Reyes Gil M, Barouqa M, Szymanski J, Gonzalez-Lugo JD, Rahman S, Billett HH. Assessment of Lupus Anticoagulant Positivity in $\mathrm{Pa}$ tients with Coronavirus Disease 2019 (COVID-19). JAMA Netw Open. 2020 Aug;3(8): e2017539.
16 Hamming I, Timens W, Bulthuis ML, Lely AT, Navis G, van Goor H. Tissue distribution of ACE2 protein, the functional receptor for SARS coronavirus. A first step in understanding SARS pathogenesis. J Pathol. 2004 Jun;203(2):631-7.

17 Khan M, Ibrahim RH, Siddiqi SA, Kerolos Y, Al-Kaylani MM, AlRukn SA, et al. COVID-19 and acute ischemic stroke - a case series from Dubai, UAE. Int JStroke. 2020 Aug;15(6):699700 